\title{
Inverse zero-sum problems and algebraic invariants
}

\author{
by \\ Benjamin Girard (Paris)
}

1. Introduction. Let $G$ be a finite Abelian group, written additively. We denote by $\exp (G)$ the exponent of $G$. If $G$ is cyclic of order $n$, it will be denoted by $C_{n}$. In the general case, we can decompose $G$ (see for instance [23]) as a direct product of cyclic groups $C_{n_{1}} \oplus \cdots \oplus C_{n_{r}}$ where $1<n_{1}|\ldots| n_{r} \in \mathbb{N}$.

In this paper, any finite sequence $S=\left(g_{1}, \ldots, g_{l}\right)$ of $l$ elements from $G$ will be called a sequence in $G$ with length $|S|=l$. Given a sequence $S=\left(g_{1}, \ldots, g_{l}\right)$ in $G$, we say that $s \in G$ is a subsum of $S$ when

$$
s=\sum_{i \in I} g_{i} \quad \text { for some } \emptyset \varsubsetneqq I \subseteq\{1, \ldots, l\} .
$$

If 0 is not a subsum of $S$, we say that $S$ is a zero-sumfree sequence. If $\sum_{i=1}^{l} g_{i}=0$, then $S$ is said to be a zero-sum sequence. If moreover $\sum_{i \in I} g_{i}$ $\neq 0$ for all proper subsets $\emptyset \subsetneq I \subsetneq\{1, \ldots, l\}$, then $S$ is called a minimal zero-sum sequence.

In a finite Abelian group $G$, the order of an element $g$ will be written $\operatorname{ord}(g)$ and for every divisor $d$ of the exponent of $G$, we denote by $G_{d}$ the subgroup of $G$ consisting of all elements of order dividing $d$ :

$$
G_{d}=\{x \in G \mid d x=0\} .
$$

For every divisor $d$ of $\exp (G)$, and every sequence $S$ in $G$, we denote by $\alpha_{d}$ the number of elements, counted with multiplicity, contained in $S$ and of order $d$. Although $\alpha_{d}$ clearly depends on $S$, we will not emphasize this dependence in the present paper, since there will be no risk of confusion.

Let $\mathcal{P}$ be the set of prime numbers. Given $n \in \mathbb{N}^{*}=\mathbb{N} \backslash\{0\}$, we denote by $\mathcal{D}_{n}$ the set of positive divisors of $n$, and we set $\tau(n)=\left|\mathcal{D}_{n}\right|$. If $n>1$, we denote by $P^{-}(n)$ the smallest prime element of $\mathcal{D}_{n}$, and we put by convention $P^{-}(1)=1$. For every $p \in \mathcal{P}, \nu_{p}(n)$ will denote the $p$-adic valuation of $n$.

2000 Mathematics Subject Classification: 11R27, 11B75, 11P99, 20D60, 20K01, 05E99, $13 \mathrm{~F} 05$.

Key words and phrases: zero-sumfree sequence, Davenport constant, cross number. 
Let $G \simeq C_{n_{1}} \oplus \cdots \oplus C_{n_{r}}$, with $1<n_{1}|\ldots| n_{r} \in \mathbb{N}$, be a finite Abelian group. We set

$$
\mathrm{D}^{*}(G)=\sum_{i=1}^{r}\left(n_{i}-1\right)+1 \quad \text { and } \quad \mathrm{d}^{*}(G)=\mathrm{D}^{*}(G)-1 .
$$

Let $\mathrm{D}(G)$ denote the smallest integer $t \in \mathbb{N}^{*}$ such that every sequence $S$ in $G$ with $|S| \geq t$ contains a non-empty zero-sum subsequence. The number $\mathrm{D}(G)$ is called the Davenport constant of the group $G$.

We denote by $\mathrm{d}(G)$ the greatest length of a zero-sumfree sequence in $G$. It can be readily seen that

$$
\mathrm{d}(G)=\mathrm{D}(G)-1 .
$$

If $G \simeq C_{\nu_{1}} \oplus \cdots \oplus C_{\nu_{s}}$, with $\nu_{i}>1$ for all $i \in \llbracket 1, s \rrbracket$, is the longest possible decomposition of $G$ into a direct product of cyclic groups, then we set

$$
\mathrm{k}^{*}(G)=\sum_{i=1}^{s} \frac{\nu_{i}-1}{\nu_{i}} .
$$

The cross number of a sequence $S=\left(g_{1}, \ldots, g_{l}\right)$, denoted by $\mathrm{k}(S)$, is then defined by

$$
\mathrm{k}(S)=\sum_{i=1}^{l} \frac{1}{\operatorname{ord}\left(g_{i}\right)} .
$$

The notion of cross number was introduced by U. Krause [17] (see also [18]). Finally, we define the so-called little cross number $\mathrm{k}(G)$ of $G$ :

$$
\mathrm{k}(G)=\max \{\mathrm{k}(S) \mid S \text { a zero-sumfree sequence in } G\} .
$$

Two elementary constructions (see [11, Proposition 5.1.8]) give the following lower bounds:

$$
\mathrm{D}^{*}(G) \leq \mathrm{D}(G) \quad \text { and } \quad \mathrm{k}^{*}(G) \leq \mathrm{k}(G) .
$$

The invariants $\mathrm{D}(G)$ and $\mathrm{k}(G)$ play a key rôle in the theory of non-unique factorization (see for instance Chapter 9 in [20], the book [11] which presents various aspects of the theory, and also the survey [12]). They have been extensively studied during the last decades and even if numerous results were proved (see Chapter 5 of [11], [7] for a survey with many references, and [14] for recent results on the cross number of finite Abelian groups), their exact values are known for very special types of groups only. We will need some of these values for finite Abelian p-groups and finite Abelian groups of rank two, so we gather them in the following theorem (see [10], [21] and [22]). 
THEOREM 1.1.

(i) Let $p \in \mathcal{P}, r \in \mathbb{N}^{*}$ and $a_{1} \leq \cdots \leq a_{r}$, where $a_{i} \in \mathbb{N}^{*}$ for all $i \in \llbracket 1, r \rrbracket$. Then, for the p-group $G \simeq C_{p^{a_{1}}} \oplus \cdots \oplus C_{p^{a_{r}}}$, we have

$$
\mathrm{D}(G)=\sum_{i=1}^{r}\left(p^{a_{i}}-1\right)+1=\mathrm{D}^{*}(G), \quad \mathrm{k}(G)=\sum_{i=1}^{r} \frac{p^{a_{i}}-1}{p^{a_{i}}}=\mathrm{k}^{*}(G) .
$$

(ii) For every $m, n \in \mathbb{N}^{*}$,

$$
\mathrm{D}\left(C_{m} \oplus C_{m n}\right)=m+m n-1=\mathrm{D}^{*}\left(C_{m} \oplus C_{m n}\right) .
$$

In particular, $\mathrm{D}\left(C_{n}\right)=n$.

The aim of this paper is to study some inverse zero-sum problems of a special type. Instead of trying to characterize explicitly, given a finite Abelian group, the structure of long zero-sumfree sequences (see [5], [3], [9], [25] and [8]), or the structure of zero-sumfree sequences with large cross number (see [13]), we study to what extent a zero-sumfree sequence can be extremal in both directions simultaneously. For instance, what is the maximal cross number of a long zero-sumfree sequence? Regarding this problem, we propose the following general conjecture.

Conjecture 1.2. Let $G \simeq C_{n_{1}} \oplus \cdots \oplus C_{n_{r}}$, with $1<n_{1}|\ldots| n_{r} \in \mathbb{N}$, be a finite Abelian group. If $S$ is a zero-sumfree sequence in $G$ with $|S| \geq \mathrm{d}^{*}(G)$, then

$$
\mathrm{k}(S) \leq \sum_{i=1}^{r} \frac{n_{i}-1}{n_{i}}
$$

In particular, $\mathrm{k}(S)<r$.

Conjecture 1.2 is closely related to the distribution of the orders of elements in a long zero-sumfree sequence. We will see that when the conjecture holds, it provides useful information on this question. In the following proposition, we gather what is currently known, to the best of our knowledge, on the structure of long zero-sumfree sequences in finite Abelian groups of rank two. This result, due to W. Gao and A. Geroldinger, can be found under a slightly different form in [11, Proposition 5.8.4].

Proposition 1.3. Let $G \simeq C_{m} \oplus C_{m n}$, where $m, n \in \mathbb{N}^{*}$, be a finite Abelian group of rank two. For every zero-sumfree sequence $S$ in $G$ with $|S|=\mathrm{d}(G)=m+m n-2$, the following two statements hold.

(i) $m|\operatorname{ord}(g)| m n$ for every $g \in S$.

(ii) The sequence $S$ contains at least

$$
m+m n-n\left(\frac{2 m-2}{P^{-}(n)}+1\right)-1 \geq m-1
$$

elements of order mn. 
The problem of the exact structure of a long zero-sumfree sequence in groups of the form $G \simeq C_{m} \oplus C_{m n}$ is also closely related to an important conjecture in additive group theory, which bears upon the so-called Property B. Let $n \geq 2$ be an integer. We say that $n$ has Property $B$ if every zero-sumfree sequence in $G \simeq C_{n} \oplus C_{n}$ with $|S|=\mathrm{d}(G)=2 n-2$ contains some element repeated at least $n-2$ times.

Property B was introduced and first studied in [4] (see also [11, Section 5.8], [19] and [9]). It is conjectured that every integer $n \geq 2$ has Property B, and recently, it was proved that an integer $n \geq 2$ has Property $\mathrm{B}$ if each prime divisor of $n$ has this property (see [6, Section 8], and [8]). Therefore, it remains to solve this problem for prime numbers. Regarding this, it can be shown that Property B holds for $n=2,3,5,7$ (see [6, Proposition 4.2]), for $n=11,13,17,19$ (see [1]) and consequently for every integer $n$ representable as a product of these numbers.

Moreover, W. Schmid [25] proved that if some integer $m \geq 2$ has Property $\mathrm{B}$, then the zero-sumfree sequences in $G \simeq C_{m} \oplus C_{m n}$ with length $\mathrm{d}(G)=m+m n-2$ can be characterized explicitly for all $n \in \mathbb{N}^{*}$. This result provides a unified way to prove Theorem 3.3 of [5] and the Theorem of [3]. It also implies, assuming that Property B holds for every integer $n \geq 2$, that Conjecture 1.2 holds true for every finite Abelian group of rank two.

2. New results and plan of the paper. In this article, we prove that Conjecture 1.2 holds for several types of finite Abelian groups. First, in Section 3, we prove some consequences of this conjecture. For instance, Conjecture 1.2 would imply two classical and long-standing conjectures related to the Davenport constant of finite Abelian groups of the form $C_{n}^{r}$.

Proposition 2.1. Let $n, r \in \mathbb{N}^{*}$ be such that Conjecture 1.2 holds for the group $C_{n}^{r}$. Then

$$
\mathrm{D}\left(C_{n}^{r}\right)=r(n-1)+1 .
$$

Moreover, every zero-sumfree sequence $S$ in $C_{n}^{r}$ with $|S|=\mathrm{d}\left(C_{n}^{r}\right)=r(n-1)$ consists only of elements of order $n$.

More generally, Conjecture 1.2 would provide the following upper bound for the Davenport constant of a finite Abelian group.

Proposition 2.2. Suppose that Conjecture 1.2 holds for $G \simeq C_{n_{1}} \oplus$ $\cdots \oplus C_{n_{r}}$ with $1<n_{1}|\ldots| n_{r} \in \mathbb{N}$. Then

$$
\mathrm{D}(G) \leq \sum_{i=1}^{r} \frac{n_{r}}{n_{i}}\left(n_{i}-1\right)+1=\mathrm{D}^{*}(G)+\sum_{i=1}^{r}\left(\frac{n_{r}}{n_{i}}-1\right)\left(n_{i}-1\right) .
$$

In Section 3, we also prove that Conjecture 1.2 holds true for finite cyclic groups and finite Abelian $p$-groups. 
Proposition 2.3. Conjecture 1.2 holds whenever:

(i) $G$ is a finite cyclic group,

(ii) $G$ is a finite Abelian p-group.

In Section 4, we present a general method, introduced in [14] to study the cross number of finite Abelian groups. Using this method, we prove in Section 5 two lemmas, which will be useful in the special case of finite Abelian groups of rank two.

In Section 6, we prove the two main theorems of this paper. The first one states that Conjecture 1.2 holds for every finite Abelian group of rank two. As already mentioned in Section 1, this result supports Property B (see [25]).

Theorem 2.4. Let $G \simeq C_{m} \oplus C_{m n}$, where $m, n \in \mathbb{N}^{*}$, be a finite Abelian group of rank two. For every zero-sumfree sequence $S$ in $G$ with $|S| \geq$ $\mathrm{d}^{*}(G)=m+m n-2$,

$$
\mathrm{k}(S) \leq \frac{m-1}{m}+\frac{m n-1}{m n} .
$$

In particular, $\mathrm{k}(S)<2$.

The second theorem, which is proved in Section 6 as well, is an effective result which states that, in a finite Abelian group of rank two, most of the elements of a long zero-sumfree sequence must have maximal order. This improves significantly the statement of Proposition 1.3(ii).

Theorem 2.5. Let $G \simeq C_{m} \oplus C_{m n}$, where $m, n \in \mathbb{N}^{*}$. For every zerosumfree sequence $S$ in $G$ with $|S|=\mathrm{d}(G)=m+m n-2$, the following two statements hold.

(i) If $n$ is a prime power, then $S$ contains at least $m n-1$ elements of order $m n$.

(ii) If $n$ is not a prime power, then $S$ contains at least

$$
\left\lceil\frac{4}{5} m n+\frac{n-5}{5}\right\rceil
$$

elements of order $m n$.

It may be observed that for every group $G \simeq C_{m} \oplus C_{m n}$, where $m, n \in$ $\mathbb{N}^{*}$ and $n \geq 2$, there exists a zero-sumfree sequence $S$ in $G$ with $|S|=$ $\mathrm{d}(G)=m+m n-2$, and which does not contain more than $m n-1$ elements of order $m n$. Indeed, let $\left(e_{1}, e_{2}\right)$ be a basis of $G$ with $\operatorname{ord}\left(e_{1}\right)=m$ and $\operatorname{ord}\left(e_{2}\right)=m n$. Then it suffices to consider the zero-sumfree sequence $S$ consisting of $e_{1}$ repeated $m-1$ times and $e_{2}$ repeated $m n-1$ times. From this point of view, Theorem 2.5 proves to be "nearly optimal". In addition, the general method presented in Section 4 can be successfully used to prove an analogue of Theorem 2.5 in the case of finite Abelian $p$-groups (see [15]). 
Finally, in Section 7, we will present and discuss a general conjecture concerning the maximal possible length of a zero-sumfree sequence with large cross number, which can be seen as a dual version of Conjecture 1.2.

3. Proofs of Propositions 2.1-2.3. We first prove the two corollaries of Conjecture 1.2 announced in Section 2.

Proof of Proposition 2.1. Let $S$ be a zero-sumfree sequence in $G \simeq C_{n}^{r}$ with maximal length $|S|=\mathrm{d}(G)=\mathrm{D}(G)-1$. Then

$$
\frac{\mathrm{D}(G)-1}{n}=\frac{|S|}{n} \leq \mathrm{k}(S) \leq r \frac{n-1}{n},
$$

which implies that $\mathrm{D}(G) \leq r(n-1)+1=\mathrm{D}^{*}(G)$, and since $\mathrm{D}^{*}(G) \leq \mathrm{D}(G)$ always holds, the equality follows. Consequently,

$$
\mathrm{k}(S)=r \frac{n-1}{n}=\frac{\mathrm{D}(G)-1}{n}=\frac{|S|}{n},
$$

and so every element $g$ of $S$ satisfies $\operatorname{ord}(g)=\exp (G)=n$.

Proof of Proposition 2.2. Let $S$ be a zero-sumfree sequence in $G \simeq C_{n_{1}} \oplus$ $\cdots \oplus C_{n_{r}}$ with $1<n_{1}|\ldots| n_{r} \in \mathbb{N}$ such that $|S|=\mathrm{d}(G)=\mathrm{D}(G)-1$. Then

$$
\frac{\mathrm{D}(G)-1}{n_{r}}=\frac{|S|}{n_{r}} \leq \mathrm{k}(S) \leq \sum_{i=1}^{r} \frac{n_{i}-1}{n_{i}},
$$

which implies the desired result.

We now prove that Conjecture 1.2 holds true for finite cyclic groups and finite Abelian $p$-groups.

Proof of Proposition 2.3. (i) Let $n \geq 2$ be an integer and let $S$ be a zero-sumfree sequence in $C_{n}$ with $|S| \geq \mathrm{d}^{*}\left(C_{n}\right)=n-1$. Then it is wellknown (see for instance [11, Theorem 5.1.10(i)]) that there exists $g \in C_{n}$ with $\operatorname{ord}(g)=n$ such that

$$
S=(\underbrace{g, \ldots, g}_{n-1 \text { times }})
$$

Consequently,

$$
\mathrm{k}(S)=\frac{n-1}{n},
$$

which gives the desired result.

(ii) Let $p \in \mathcal{P}, r \in \mathbb{N}^{*}$, and $G \simeq C_{p^{a_{1}}} \oplus \cdots \oplus C_{p^{a_{r}}}$ with $a_{1} \leq \cdots \leq a_{r}$ and $a_{i} \in \mathbb{N}^{*}$ for all $i \in \llbracket 1, r \rrbracket$. By Theorem 1.1(i),

$$
\mathrm{k}(G)=\sum_{i=1}^{r} \frac{p^{a_{i}}-1}{p^{a_{i}}}=\mathrm{k}^{*}(G) .
$$


Thus, for every zero-sumfree sequence $S$ in $G$, in particular for those with $|S| \geq \mathrm{d}^{*}(G)$, one indeed has, by the very definition of the little cross number,

$$
\mathrm{k}(S) \leq \mathrm{k}(G)=\sum_{i=1}^{r} \frac{p^{a_{i}}-1}{p^{a_{i}}},
$$

and the proof is complete.

4. Outline of a new method. Let $G$ be a finite Abelian group, and let $S$ be a sequence of elements in $G$. The general method that we will use in this paper (see also [14] and [15] for applications in two other contexts) consists in considering, for every $d^{\prime}, d \in \mathbb{N}$ such that $1 \leq d^{\prime}|d| \exp (G)$, the following exact sequence:

$$
0 \rightarrow G_{d / d^{\prime}} \hookrightarrow G_{d} \stackrel{\pi_{\left(d^{\prime}, d\right)}}{\longrightarrow} G_{d} / G_{d / d^{\prime}} \rightarrow 0 .
$$

Now, let $U$ be the subsequence of $S$ consisting of all elements whose order divides $d$. If, for some $1 \leq d^{\prime}|d| \exp (G)$, it is possible to find sufficiently many disjoint non-empty zero-sum subsequences in $\pi_{\left(d^{\prime}, d\right)}(U)$, that is, sufficiently many disjoint subsequences in $U$ the sum of each being an element of order dividing $d / d^{\prime}$, then $S$ cannot be a zero-sumfree sequence in $G$.

To make this idea more precise, we introduced in [14] the following number, which can be seen as an extension of the classical Davenport constant.

Let $G \simeq C_{n_{1}} \oplus \cdots \oplus C_{n_{r}}$, with $1<n_{1}|\ldots| n_{r} \in \mathbb{N}$ and $d^{\prime}, d \in \mathbb{N}$ be such that $1 \leq d^{\prime}|d| \exp (G)$. We denote by $\mathrm{D}_{\left(d^{\prime}, d\right)}(G)$ the smallest $t \in \mathbb{N}^{*}$ such that every sequence $S$ in $G_{d}$ with $|S| \geq t$ contains a non-empty subsequence with sum in $G_{d / d^{\prime}}$.

Using this definition, we can prove the following simple lemma, which is an illustration of our idea. This result will be useful in Section 5 and states that given a finite Abelian group, there exist strong constraints on the way the orders of elements are distributed within a zero-sumfree sequence.

Lemma 4.1. Let $G$ be a finite Abelian group and let $d^{\prime}, d \in \mathbb{N}$ be such that $1 \leq d^{\prime}|d| \exp (G)$. Given a sequence $S$ of elements in $G$, write $T$ for the subsequence of $S$ consisting of all elements whose order divides $d / d$, and write $U$ for the subsequence of $S$ consisting of all elements whose order divides $d$ (in particular, $T \subseteq U$ ). Then $S$ is not a zero-sumfree sequence whenever

$$
|T|+\left\lfloor\frac{|U|-|T|}{\mathrm{D}_{\left(d^{\prime}, d\right)}(G)}\right\rfloor \geq \mathrm{D}_{\left(d / d^{\prime}, d / d^{\prime}\right)}(G) .
$$

Proof. Set $\Delta=\mathrm{D}_{\left(d / d^{\prime}, d / d^{\prime}\right)}(G)$. The above inequality implies that there are $\Delta$ disjoint subsequences $S_{1}, \ldots, S_{\Delta}$ of $S$, the sum of each being an element of order dividing $d / d^{\prime}$. Then, by the very definition of $\mathrm{D}_{\left(d / d^{\prime}, d / d^{\prime}\right)}(G)$, $S$ has to contain a non-empty zero-sum subsequence. 
Now, in order to obtain effective inequalities from the symbolic constraints of Lemma 4.1, one can use a result proved in [14], which states that for any finite Abelian group $G$ and every $1 \leq d^{\prime}|d| \exp (G)$, the invariant $\mathrm{D}_{\left(d^{\prime}, d\right)}(G)$ is linked with the classical Davenport constant of a particular subgroup of $G$, which can be characterized explicitly. In order to define this subgroup properly, we introduce the following notation.

For all $i \in \llbracket 1, r \rrbracket$, we set

$$
A_{i}=\operatorname{gcd}\left(d^{\prime}, n_{i}\right), \quad B_{i}=\frac{\operatorname{lcm}\left(d, n_{i}\right)}{\operatorname{lcm}\left(d^{\prime}, n_{i}\right)}, \quad v_{i}\left(d^{\prime}, d\right)=\frac{A_{i}}{\operatorname{gcd}\left(A_{i}, B_{i}\right)} .
$$

For instance, whenever $d$ divides $n_{i}$, we have $v_{i}\left(d^{\prime}, d\right)=\operatorname{gcd}\left(d^{\prime}, n_{i}\right)=d^{\prime}$, and in particular $v_{r}\left(d^{\prime}, d\right)=d^{\prime}$. We can now state our result on $\mathrm{D}_{\left(d^{\prime}, d\right)}(G)$ (see [14, Proposition 3.1]).

Proposition 4.2. Let $G \simeq C_{n_{1}} \oplus \cdots \oplus C_{n_{r}}$, with $1<n_{1}|\ldots| n_{r} \in \mathbb{N}$, and let $d^{\prime}, d \in \mathbb{N}$ be such that $1 \leq d^{\prime}|d| \exp (G)$. Then

$$
\mathrm{D}_{\left(d^{\prime}, d\right)}(G)=\mathrm{D}\left(C_{v_{1}\left(d^{\prime}, d\right)} \oplus \cdots \oplus C_{v_{r}\left(d^{\prime}, d\right)}\right) .
$$

5. Two lemmas related to zero-freeness in $G \simeq C_{m} \oplus C_{m n}$. In this section, we show how the method of Section 4 can be used to obtain two key lemmas for the proofs of Theorems 2.4 and 2.5. We first prove the following result.

Lemma 5.1. Let $G \simeq C_{m} \oplus C_{m n}$, where $m, n \in \mathbb{N}^{*}, n \geq 2$, and let $S$ be a zero-sumfree sequence in $G$ with $|S| \geq \mathrm{d}^{*}(G)=m+m n-2$. Then, for every $l \in \mathcal{D}_{n} \backslash\{n\}$,

$$
\sum_{d \in \mathcal{D}_{l}} \alpha_{m d} \leq m-1
$$

Proof. Let $l \in \mathcal{D}_{n} \backslash\{n\}, d^{\prime}=n / l$ and $d=m n$, which leads to $d / d^{\prime}=m l$. Set $m^{\prime}=\operatorname{gcd}\left(d^{\prime}, m\right)$, and let $T$ and $U$ be the two subsequences of $S$ defined in Lemma 4.1. In particular, $T \subseteq U=S$, and by Proposition 1.3(i),

$$
|T|=\sum_{\bar{d} \in \mathcal{D}_{l}} \alpha_{m \bar{d}} .
$$

To start with, we determine the exact value of $\mathrm{D}_{\left(d^{\prime}, d\right)}(G)$. One has

$$
v_{1}\left(d^{\prime}, d\right)=\frac{m^{\prime}}{\operatorname{gcd}\left(m^{\prime}, \frac{\operatorname{lcm}(d, m)}{\operatorname{lcm}\left(d^{\prime}, m\right)}\right)}=\frac{m^{\prime}}{\operatorname{gcd}\left(m^{\prime}, \frac{d}{d^{\prime}} \frac{m^{\prime}}{m}\right)}=\frac{m^{\prime}}{\operatorname{gcd}\left(m^{\prime}, m^{\prime} l\right)}=1,
$$

and since $v_{2}\left(d^{\prime}, d\right)=d^{\prime}$, Proposition 4.2 and Theorem 1.1(ii) yield

$$
\mathrm{D}_{\left(d^{\prime}, d\right)}(G)=\mathrm{D}\left(C_{v_{1}\left(d^{\prime}, d\right)} \oplus C_{v_{2}\left(d^{\prime}, d\right)}\right)=\mathrm{D}\left(C_{n / l}\right)=n / l .
$$


Now, suppose that $|T| \geq m$. Since $l \in \mathcal{D}_{n} \backslash\{n\}$, we obtain

$$
\begin{aligned}
|T|+\frac{|U|-|T|}{\mathrm{D}_{\left(d^{\prime}, d\right)}(G)} & \geq|T|+\frac{l(m+m n-2-|T|)}{n} \geq m+\frac{l(m n-2)}{n} \\
& =(m+m l-1)-\frac{l}{n}+\frac{n-l}{n} \\
& >(m+m l-1)-\frac{l}{n}=\mathrm{D}_{\left(d / d^{\prime}, d / d^{\prime}\right)}(G)-\frac{1}{\mathrm{D}_{\left(d^{\prime}, d\right)}(G)},
\end{aligned}
$$

and, according to Lemma 4.1, $S$ must contain a non-empty zero-sum subsequence, which is a contradiction. Thus, $|T| \leq m-1$, as desired.

Now, let $n \geq 2$ be an integer, and $p_{1}, \ldots, p_{r}$ be its distinct prime divisors. Given $m \in \mathbb{N}^{*}$ and a zero-sumfree sequence $S$ in $G \simeq C_{m} \oplus C_{m n}$ with $|S| \geq \mathrm{d}^{*}(G)=m+m n-2$, Lemma 5.1 implies that the integers $\alpha_{m d} \in \mathbb{N}$, where $d \in \mathcal{D}_{n} \backslash\{n\}$, satisfy the following $r$ linear constraints:

$$
\sum_{d \in \mathcal{D}_{n / p_{i}}} \alpha_{m d} \leq m-1 \quad \text { for all } i \in \llbracket 1, r \rrbracket .
$$

In the next lemma, we solve a linear integer programming problem on the divisor lattice of $n$, in order to obtain the maximum value of the function

$$
\left(\alpha_{m d}\right)_{d \in \mathcal{D}_{n} \backslash\{n\}} \mapsto \sum_{d \in \mathcal{D}_{n} \backslash\{n\}} \frac{\alpha_{m d}}{d}
$$

under the above $r$ constraints (the reader interested in linear programming methods is referred to the book [26] for an exhaustive presentation of the subject).

Lemma 5.2. Let $m, n \in \mathbb{N}^{*}$ with $n \geq 2$, and let $\left(x_{d}\right)_{d \in \mathcal{D}_{n} \backslash\{n\}}$ be a sequence of positive integers such that for every prime divisor $p$ of $n$, one has the following linear constraint:

$$
\sum_{d \in \mathcal{D}_{n / p}} x_{d} \leq m-1 .
$$

Then one has the following inequality, which is best possible:

$$
\sum_{d \in \mathcal{D}_{n} \backslash\{n\}} \frac{x_{d}}{d} \leq m-1 .
$$

Proof. Let $p_{1}, \ldots, p_{r}$ be the distinct prime divisors of $n$. For every $k \in$ $\llbracket 0, m-1 \rrbracket$, let $\mathcal{S}_{k}$ be the set of all sequences $x=\left(x_{d}\right)_{d \in \mathcal{D}_{n} \backslash\{n\}}$ of positive integers which satisfy the above linear constraints and have $x_{1}=m-k-1$. Now, we prove, by induction on $k \in \llbracket 0, m-1 \rrbracket$, that

$$
\sum_{d \in \mathcal{D}_{n} \backslash\{n\}} \frac{x_{d}}{d} \leq m-1 \quad \text { for every } x \in \mathcal{S}_{k} .
$$


If $k=0$, then for every $x \in \mathcal{S}_{0}$, the linear constraints imply that $x_{d}=0$ for all $d \in \mathcal{D}_{n} \backslash\{1, n\}$, which gives

$$
\sum_{d \in \mathcal{D}_{n} \backslash\{n\}} \frac{x_{d}}{d}=m-1 .
$$

Assume now that the inequality is valid for $k-1 \geq 0$. Define

$$
f: \mathcal{D}_{n} \backslash\{n\} \rightarrow\{\mathcal{A} \mid \emptyset \varsubsetneqq \mathcal{A} \subseteq \llbracket 1, r \rrbracket\}, \quad d \mapsto\left\{i \in \llbracket 1, r \rrbracket \mid d \in \mathcal{D}_{n / p_{i}}\right\} .
$$

Let $x \in \mathcal{S}_{k}$ and let $\mathcal{L}$ be the set of $d \in \mathcal{D}_{n} \backslash\{1, n\}$ such that $x_{d} \geq 1$. By definition, for every $d \in \mathcal{D}_{n} \backslash\{n\},|f(d)|$ is the number of linear constraints in which the variable $x_{d}$ appears. Thus, for every prime divisor $p$ of $n, x_{n / p}$ appears in only one linear constraint, and we may assume, without loss of generality, that

$$
\sum_{d \in \mathcal{D}_{n / p}} x_{d}=m-1
$$

Hence, for every $i \in \llbracket 1, r \rrbracket$, the set $\mathcal{L} \cap \mathcal{D}_{n / p_{i}}$ is non-empty, and so

$$
\bigcup_{d \in \mathcal{L}} f(d)=\llbracket 1, r \rrbracket .
$$

Let $\mathcal{L}^{\prime}$ be a subset of $\mathcal{L}$ of minimal cardinality such that

$$
\bigcup_{d \in \mathcal{L}^{\prime}} f(d)=\llbracket 1, r \rrbracket .
$$

Since $f(d)$ is a non-empty set for every $d \in \mathcal{D}_{n} \backslash\{n\}$,

$$
\bigcup_{d \in \mathcal{L}^{\prime \prime}} f(d) \subsetneq \llbracket 1, r \rrbracket \quad \text { for all } \emptyset \subsetneq \mathcal{L}^{\prime \prime} \subsetneq \mathcal{L}^{\prime}
$$

Now, one can notice the following two facts.

FACT 1. For every $d \in \mathcal{L}^{\prime}$, one has $f(d) \leq r-\left|\mathcal{L}^{\prime}\right|+1$, and in particular, $\left|\mathcal{L}^{\prime}\right| \leq r$.

This is a straightforward consequence of the following combinatorial lemma.

Lemma 5.3. Let $r \in \mathbb{N}^{*}$ and $\mathcal{A}_{1}, \ldots, \mathcal{A}_{s}$ be non-empty subsets of $\llbracket 1, r \rrbracket$ such that

$$
\bigcup_{i \in \llbracket 1, s \rrbracket} \mathcal{A}_{i}=\llbracket 1, r \rrbracket \quad \text { and } \quad \bigcup_{i \in I} \mathcal{A}_{i} \subsetneq \llbracket 1, r \rrbracket \quad \text { for any } \emptyset \subsetneq I \subsetneq \llbracket 1, s \rrbracket .
$$

Then $\left|\mathcal{A}_{i}\right| \leq r-s+1$ for all $i \in \llbracket 1, s \rrbracket$.

Proof. By symmetry, it suffices to prove that $\left|\mathcal{A}_{1}\right| \leq r-s+1$. Assume to the contrary that $\left|\mathcal{A}_{1}\right| \geq r-s+2$. Since, for all $i \in \llbracket 1, s-1 \rrbracket$, the set $\mathcal{A}_{i+1}$ 
must contain at least one element from $\llbracket 1, r \rrbracket \backslash\left(\mathcal{A}_{1} \cup \cdots \cup \mathcal{A}_{i}\right)$, one obtains the following inequality:

$$
\left|\mathcal{A}_{1} \cup \cdots \cup \mathcal{A}_{i+1}\right| \geq\left|\mathcal{A}_{1} \cup \cdots \cup \mathcal{A}_{i}\right|+1 .
$$

Therefore, by an easy induction argument,

$$
\left|\mathcal{A}_{1} \cup \cdots \cup \mathcal{A}_{s-1}\right| \geq(r-s+2)+(s-2)=r,
$$

and so $\mathcal{A}_{1} \cup \cdots \cup \mathcal{A}_{s-1}=\llbracket 1, r \rrbracket$, which is a contradiction.

FACT 2. For every $d \in \mathcal{D}_{n} \backslash\{n\}$,

$$
d \geq \min f^{-1}(f(d)) \geq \prod_{i \in \llbracket 1, r \rrbracket \backslash f(d)} p_{i}^{\nu_{p_{i}}(n)} \geq 2^{r-|f(d)|} .
$$

Now, using Facts 1 and 2, we can prove the desired result, by considering the sequence $y=\left(y_{d}\right)_{d \in \mathcal{D}_{n} \backslash\{n\}}$ obtained from $x$ in the following way:

$$
y_{d}= \begin{cases}x_{1}+1 & \text { if } d=1 \\ x_{d}-1 & \text { if } d \in \mathcal{L}^{\prime} \\ x_{d} & \text { otherwise. }\end{cases}
$$

It is readily seen that $y \in \mathcal{S}_{k-1}$. Therefore, Facts 1 and 2 give the following inequalities:

$$
\begin{aligned}
m-1 & \geq \sum_{d \in \mathcal{D}_{n} \backslash\{n\}} \frac{y_{d}}{d}=\left(\sum_{d \in \mathcal{D}_{n} \backslash\{n\}} \frac{x_{d}}{d}\right)+\left(1-\sum_{d \in \mathcal{L}^{\prime}} \frac{1}{d}\right) \\
& \geq\left(\sum_{d \in \mathcal{D}_{n} \backslash\{n\}} \frac{x_{d}}{d}\right)+\left(1-\sum_{d \in \mathcal{L}^{\prime}} \frac{1}{2^{r-|f(d)|}}\right) \\
& \geq\left(\sum_{d \in \mathcal{D}_{n} \backslash\{n\}} \frac{x_{d}}{d}\right)+\left(1-\sum_{d \in \mathcal{L}^{\prime}} \frac{1}{2^{\left|\mathcal{L}^{\prime}\right|-1}}\right) \\
& \geq\left(\sum_{d \in \mathcal{D}_{n} \backslash\{n\}} \frac{x_{d}}{d}\right)+\left(1-\frac{\left|\mathcal{L}^{\prime}\right|}{2^{\left|\mathcal{L}^{\prime}\right|-1}}\right) \geq \sum_{d \in \mathcal{D}_{n} \backslash\{n\}} \frac{x_{d}}{d},
\end{aligned}
$$

which completes the proof.

6. Proofs of the two main theorems. We first show that every finite Abelian group of rank two satisfies Conjecture 1.2. The proof is a direct application of Lemmas 5.1 and 5.2.

Proof of Theorem 2.4. Let $G$ and $S$ be as in the statement of the theorem, in particular $|S| \geq \mathrm{d}^{*}(G)=m+m n-2$. Since $\mathrm{d}(G)=\mathrm{d}^{*}(G)$ by Theorem 1.1(ii), we obtain $|S|=\mathrm{d}^{*}(G)=m+m n-2$.

If $n=1$, then the desired result follows directly from Proposition 1.3(i), since every element of $S$ has order $m$. Now, suppose that $n \geq 2$. Using 
Proposition 1.3(i), we obtain

$$
\mathrm{k}(S)=\sum_{d \in \mathcal{D}_{m n}} \frac{\alpha_{d}}{d}=\sum_{d \in \mathcal{D}_{n}} \frac{\alpha_{m d}}{m d},
$$

and we can distinguish two cases.

CASE 1: $\alpha_{m n} \geq m n-1$. In this case, applying Proposition 1.3(i), one obtains

$$
\sum_{d \in \mathcal{D}_{n} \backslash\{n\}} \alpha_{m d}=|S|-\alpha_{m n}
$$

which implies

$$
\begin{aligned}
\mathrm{k}(S) & =\sum_{d \in \mathcal{D}_{n} \backslash\{n\}} \frac{\alpha_{m d}}{m d}+\frac{\alpha_{m n}}{m n} \leq \frac{|S|-\alpha_{m n}}{m}+\frac{\alpha_{m n}}{m n} \\
& \leq \frac{|S|-(m n-1)}{m}+\frac{m n-1}{m n}=\frac{m-1}{m}+\frac{m n-1}{m n} .
\end{aligned}
$$

CASE 2: $\alpha_{m n} \leq m n-1$. Then, by Lemmas 5.1 and 5.2 , we obtain

$$
\mathrm{k}(S)=\sum_{d \in \mathcal{D}_{n} \backslash\{n\}} \frac{\alpha_{m d}}{m d}+\frac{\alpha_{m n}}{m n} \leq \frac{m-1}{m}+\frac{\alpha_{m n}}{m n} \leq \frac{m-1}{m}+\frac{m n-1}{m n},
$$

which completes the proof.

Now, we prove Theorem 2.5, which gives a lower bound for the number of elements with maximal order in a long zero-sumfree sequence of a finite Abelian group of rank two.

Proof of Theorem 2.5. Let $G$ and $S$ be as in the statement.

(i) Let $p \in \mathcal{P}$ and $a \in \mathbb{N}$ be such that $n=p^{a}$. If $a=0$, then $G \simeq C_{m} \oplus C_{m}$ and, by Proposition 1.3(i), every element of $S$ has order $m$. Now, suppose that $a \geq 1$. Then, by Lemma 5.1 ,

$$
|S|-\alpha_{m n}=\sum_{d \in \mathcal{D}_{p^{a}-1}} \alpha_{m d} \leq m-1,
$$

which indeed implies that

$$
\alpha_{m n} \geq|S|-(m-1)=m+m n-2-(m-1)=m n-1 .
$$

(ii) If $\tau(n) \leq 3$, then $n$ has to be a prime power, and the result follows by (i). Now, suppose that $\mathcal{D}_{n}=\left\{d_{0}=1<d_{1}<d_{2}<d_{3}<\cdots\right\}$ contains at least four elements. In particular, $n \geq 6$. By Lemmas 5.1 and 5.2,

$$
\sum_{d \in \mathcal{D}_{n} \backslash\{n\}} \frac{\alpha_{m d}}{m d} \leq \frac{m-1}{m},
$$


that is,

$$
d_{1} \frac{\alpha_{m n / d_{1}}}{m n}+d_{2} \frac{\alpha_{m n / d_{2}}}{m n}+d_{3} \frac{\alpha_{m n / d_{3}}}{m n}+\sum_{\substack{d \in \mathcal{D}_{n} \\ d>d_{3}}} d \frac{\alpha_{m n / d}}{m n} \leq \frac{m-1}{m} .
$$

Now, we can distinguish two cases.

CASE 1: $d_{3}=4$. Then $d_{1}=2, d_{2}=3$ and (1) implies

$$
2 \frac{\alpha_{m n / 2}}{m n}+3 \frac{\alpha_{m n / 3}}{m n}+4 \frac{\alpha_{m n / 4}}{m n}+5 \sum_{\substack{d \in \mathcal{D}_{n} \\ d>d_{3}}} \frac{\alpha_{m n / d}}{m n} \leq \frac{m-1}{m} .
$$

But since

$$
\sum_{\substack{d \in \mathcal{D}_{n} \\ d>d_{3}}} \alpha_{m n / d}=|S|-\alpha_{m n}-\alpha_{m n / 2}-\alpha_{m n / 3}-\alpha_{m n / 4},
$$

relation (2) implies

$$
5 \frac{m+m n-2-\alpha_{m n}}{m n}-3 \frac{\alpha_{m n / 2}}{m n}-2 \frac{\alpha_{m n / 3}}{m n}-\frac{\alpha_{m n / 4}}{m n} \leq \frac{m-1}{m},
$$

that is,

$$
5 \frac{m+m n-2-\alpha_{m n}}{m n}-\frac{\alpha_{m n / 2}+\alpha_{m n / 4}}{m n}-2 \frac{\alpha_{m n / 2}+\alpha_{m n / 3}}{m n} \leq \frac{m-1}{m} .
$$

Now using the fact that, by Lemma 5.1,

$$
\alpha_{m n / 2}+\alpha_{m n / 4} \leq m-1 \quad \text { and } \quad \alpha_{m n / 3} \leq m-1,
$$

we obtain

$$
5 \frac{m+m n-2-\alpha_{m n}}{m n}-\frac{m-1}{m n}-2 \frac{2(m-1)}{m n} \leq \frac{m-1}{m},
$$

which is equivalent to

$$
5 \frac{m n-1-\alpha_{m n}}{m n} \leq \frac{m-1}{m}
$$

that is,

$$
5(m n-1)-n(m-1) \leq 5 \alpha_{m n},
$$

and thus

$$
\frac{4}{5} m n+\frac{n-5}{5} \leq \alpha_{m n}
$$

which is the desired result.

CAsE $2: d_{3} \geq 5$. Then (1) implies

$$
d_{1} \frac{\alpha_{m n / d_{1}}}{m n}+d_{2} \frac{\alpha_{m n / d_{2}}}{m n}+5 \sum_{\substack{d \in \mathcal{D}_{n} \\ d \geq d_{3}}} \frac{\alpha_{m n / d}}{m n} \leq \frac{m-1}{m}
$$


But since

$$
\sum_{\substack{d \in \mathcal{D}_{n} \\ d \geq d_{3}}} \alpha_{m n / d}=|S|-\alpha_{m n}-\alpha_{m n / d_{1}}-\alpha_{m n / d_{2}},
$$

relation (3) implies

$$
5 \frac{m+m n-2-\alpha_{m n}}{m n}+\left(d_{1}-5\right) \frac{\alpha_{m n / 2}}{m n}+\left(d_{2}-5\right) \frac{\alpha_{m n / d_{2}}}{m n} \leq \frac{m-1}{m} .
$$

Therefore, since $d_{1} \geq 2$ and $d_{2} \geq 3$, we have

$$
5 \frac{m+m n-2-\alpha_{m n}}{m n}-3 \frac{m-1}{m n}-2 \frac{m-1}{m n} \leq \frac{m-1}{m},
$$

that is,

$$
5 \frac{m n-1-\alpha_{m n}}{m n} \leq \frac{m-1}{m},
$$

which leads to

$$
\frac{4}{5} m n+\frac{n-5}{5} \leq \alpha_{m n}
$$

and the proof is complete.

7. A concluding remark. Given a finite Abelian group $G$, the investigation of the maximal possible length of a zero-sumfree sequence $S$ in $G$ with large cross number may also be of interest. Concerning this question, we propose the following general conjecture, which can be seen as a dual version of Conjecture 1.2.

Conjecture 7.1. Let $G$ be a finite Abelian group and $G \simeq C_{\nu_{1}} \oplus \cdots$ $\oplus C_{\nu_{s}}$, with $\nu_{i}>1$ for all $i \in \llbracket 1, s \rrbracket$, be its longest possible decomposition into a direct product of cyclic groups. If $S$ is a zero-sumfree sequence in $G$ such that $\mathrm{k}(S) \geq \mathrm{k}^{*}(G)$, then

$$
|S| \leq \sum_{i=1}^{s}\left(\nu_{i}-1\right) .
$$

It can easily be seen, by Theorem 1.1(i), that Conjecture 7.1 holds true for finite Abelian $p$-groups. Even in the case of finite cyclic groups which are not $p$-groups, this problem is still wide open. Yet, in this special case, the following result supports the idea that a zero-sumfree sequence with large cross number has to be "short".

TheOREM 7.2. Suppose that $n \in \mathbb{N}^{*}$ is not a prime power, and let $S$ be a zero-sumfree sequence in $C_{n}$ such that $\mathrm{k}(S) \geq \mathrm{k}^{*}\left(C_{n}\right)$. Then

$$
|S| \leq\lfloor n / 2\rfloor \text {. }
$$

Proof. We use the notion of index of a sequence in a finite cyclic group, introduced implicitly in [16, Conjecture, p. 344], and more explicitly in [2]. 
Let $g \in C_{n}$ with $\operatorname{ord}(g)=n$, and let $S=\left(g_{1}, \ldots, g_{l}\right)=\left(n_{1} g, \ldots, n_{l} g\right)$, where $n_{1}, \ldots, n_{l} \in \llbracket 0, n-1 \rrbracket$, be a sequence in $C_{n}$. We define

$$
\|S\|_{g}=\sum_{i=1}^{l} \frac{n_{i}}{n} .
$$

Since

$$
\frac{\operatorname{gcd}\left(n_{i}, n\right)}{n}=\frac{1}{\operatorname{ord}\left(g_{i}\right)} \quad \text { for every } i \in \llbracket 1, l \rrbracket,
$$

one can notice that $\|S\|_{g} \geq \mathrm{k}(S)$ for all $g \in C_{n}$ with $\operatorname{ord}(g)=n$. Then the index of $S$, denoted by index $(S)$, is defined by

$$
\operatorname{index}(S)=\min _{\substack{g \in C_{n} \\ \operatorname{ord}(g)=n}}\|S\|_{g}
$$

Now, if $n$ is not a prime power and $S$ is a zero-sumfree sequence in $C_{n}$ such that $\mathrm{k}(S) \geq \mathrm{k}^{*}\left(C_{n}\right)$, one obtains, by the very definition of the index,

$$
\operatorname{index}(S) \geq \mathrm{k}(S) \geq \mathrm{k}^{*}\left(C_{n}\right)>1 \text {. }
$$

Therefore, a result of Savchev and Chen (see Theorem 9 in [24]) yields $|S| \leq\lfloor n / 2\rfloor$.

In particular, Theorem 7.2 implies that Conjecture 7.1 holds true for all cyclic groups of the form $C_{2 p^{a}}$, where $p \in \mathcal{P}$ and $a \in \mathbb{N}$.

Acknowledgments. I am grateful to my Ph.D. advisor Alain Plagne for his help during the preparation of this paper. I would also like to thank Alfred Geroldinger and Wolfgang Schmid for useful remarks on a preliminary version of this work.

\section{References}

[1] G. Bhowmik, I. Halupczok and J. C. Schlage-Puchta, The structure of maximal zero-sum free sequences II, submitted.

[2] S. T. Chapman, M. Freeze and W. W. Smith, Minimal zero sequences and the strong Davenport constant, Discrete Math. 203 (1999), 271-277.

[3] F. Chen and S. Savchev, Minimal zero-sum sequences of maximum length in the group $C_{3} \oplus C_{3 k}$, Integers 7 (2007), \#A42.

[4] W. Gao and A. Geroldinger, On long minimal zero sequences in finite abelian groups, Period. Math. Hungar. 38 (1999), 179-211.

[5] W. Gao and A. Geroldinger, On the order of elements in long minimal zero-sum sequences, ibid. 44 (2002), 63-73.

[6] -, 一, On zero-sum sequences in $\mathbb{Z} / n \mathbb{Z} \oplus \mathbb{Z} / n \mathbb{Z}$, Integers 3 (2003), \#A8.

[7] - - - Zero-sum problems in finite abelian groups: a survey, Expo. Math. 24 (2006), 337-369.

[8] W. Gao, A. Geroldinger and D. Grynkiewicz, Inverse zero-sum problems III, submitted. 
[9] W. Gao, A. Geroldinger and W. Schmid, Inverse zero-sum problems, Acta Arith. 128 (2007), 245-279.

[10] A. Geroldinger, The cross number of finite abelian groups, J. Number Theory 48 (1994), 219-223.

[11] A. Geroldinger and F. Halter-Koch, Non-Unique Factorizations. Algebraic, Combinatorial and Analytic Theory, Pure Appl. Math. 278, Chapman \& Hall/CRC, 2006.

[12] - - , Non-unique factorizations: a survey, in: Multiplicative Ideal Theory in Commutative Algebra, Springer, New York, 2006, 207-226.

[13] A. Geroldinger and R. Schneider, On minimal zero sequences with large cross number, Ars Combin. 46 (1997), 297-303.

[14] B. Girard, A new upper bound for the cross number of finite Abelian groups, Israel J. Math., to appear.

[15] -, Inverse zero-sum problems in finite Abelian p-groups, submitted.

[16] D. Kleitman and P. Lemke, An addition theorem on the integers modulo $n$, J. Number Theory 31 (1989), 335-345.

[17] U. Krause, A characterization of algebraic number fields with cyclic class group of prime power order, Math. Z. 186 (1984), 143-148.

[18] U. Krause and C. Zahlten, Arithmetic in Krull monoids and the cross number of divisor class groups, Mitt. Math. Ges. Hamburg 12 (1991), 681-696.

[19] G. Lettl and W. Schmid, Minimal zero-sum sequences in $C_{n} \oplus C_{n}$, Eur. J. Combin. 28 (2007), 742-753.

[20] W. Narkiewicz, Elementary and Analytic Theory of Algebraic Numbers, 3rd ed., Springer, 2004.

[21] J. E. Olson, A combinatorial problem on finite abelian groups I, J. Number Theory 1 (1969), 8-10.

[22] —, A combinatorial problem on finite abelian groups II, ibid., 195-199.

[23] P. Samuel, Théorie algébrique des nombres, Hermann, 2003.

[24] S. Savchev and F. Chen, Long zero-free sequences in finite cyclic groups, Discrete Math. 307 (2007), 2671-2679.

[25] W. Schmid, Inverse zero-sum problems II, submitted.

[26] A. Schrijver, Theory of Linear and Integer Programming, Wiley, 1998.

Centre de Mathématiques Laurent Schwartz

UMR $7640 \mathrm{du}$ CNRS

École polytechnique

91128 Palaiseau Cedex, France

E-mail: benjamin.girard@math.polytechnique.fr

Received on 4.2.2008

and in revised form on 25.6.2008 